\title{
Fault Tolerant Smart Transducer Interfaces for Safety-Critical Avionics Applications
}

Safwen Bouanen ${ }^{1}$, Claude Thibeault ${ }^{1}$, Yvon Savaria ${ }^{2}$, José-Philippe Tremblay², Guchuan Zhu²

1. École de Technologie Supérieure

2. École Polytechnique de Montréal 


\section{Outline}

1. Introduction

2. IEEE 1451

3. Interfaces design

4. The prototype

5. Test and validation

6. Conclusion 


\section{Introduction (1)}

Current trends in the avionics domain

) Ever increasing number of functions/transducers

) Information flow increase

> Different communication protocols

Diversity in the transducers market

) Migration to smart transducer interfaces

) Increasing design effort, cost and time 


\section{Introduction (2)}

) Fault tolerance challenges

- DIMA/IMA2G: I/O and computation close to transducers

- Hardware redundancy complicates fault diagnosis

- Strict reliability requirements

) Solutions: Transducer interfaces with:

- Embedded fault tolerance mechanisms

- Fail-safe capabilities

- Graceful performance degradation 


\section{IEEE 1451 Standard (1)}

> IEEE Standard for a Smart Transducer Interface for Sensors and Actuators (IEEE1451)

) Adoption Advantages

- Increased compatibility

- Independent of the selected network

- Reduced design, installation and update effort

) Considered but not yet adopted by the avionics domain

Vault tolerance aspects not yet fully addressed. 


\section{IEEE 1451 Standard (2)}

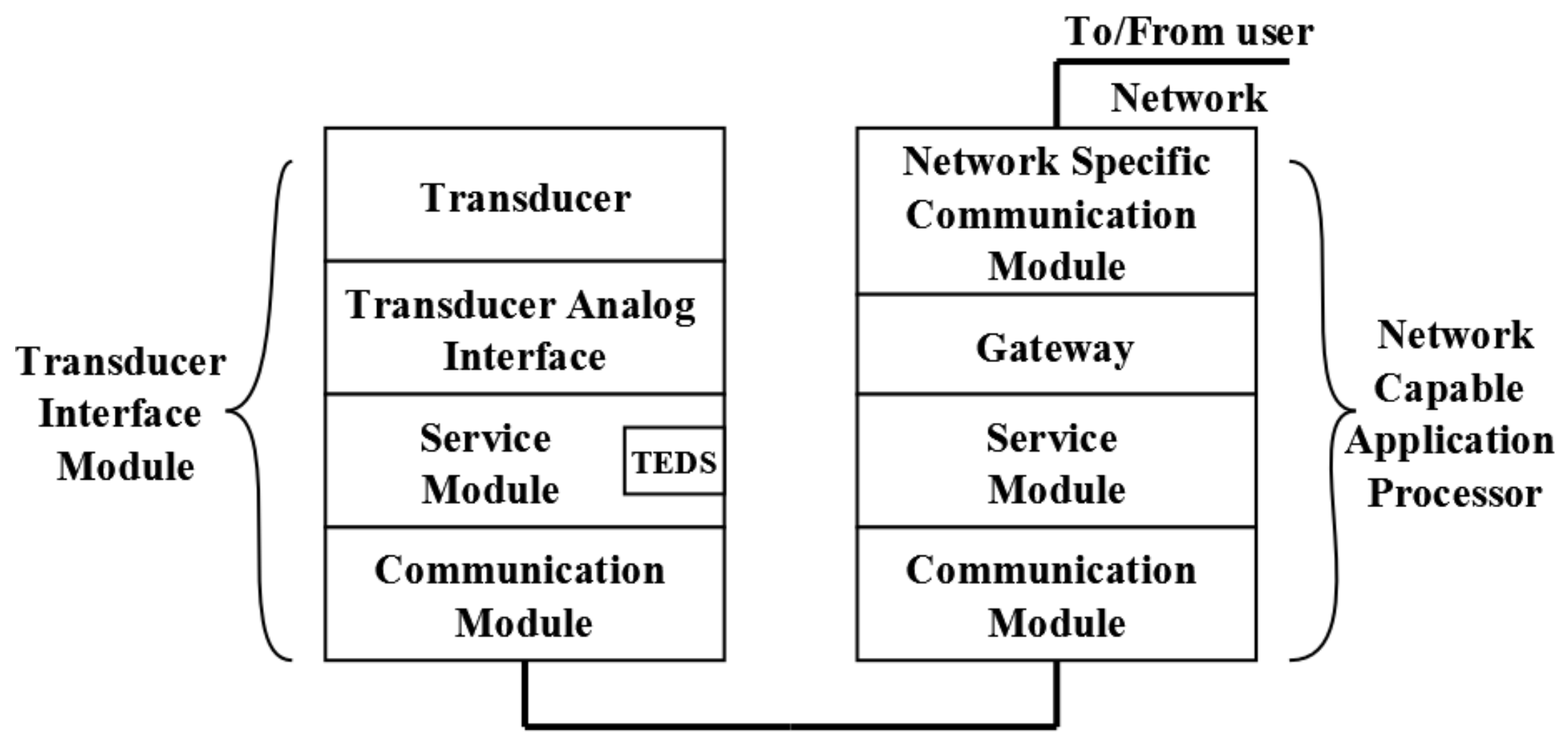

IEEE 1451 Reference Model 


\section{Interfaces Design 3.1 Requirements definition}

> The interface must be able to deal with transient and permanent faults

> The interface should fail in a safe manner

> The interface should ensure graceful degradation

) MTBF smaller than $10^{-6} /$ operating hours

Vnannunciated errors shall be less than $10^{-6} /$ operating hours 


\section{Interfaces Design 3.2 Single-chip Interface}

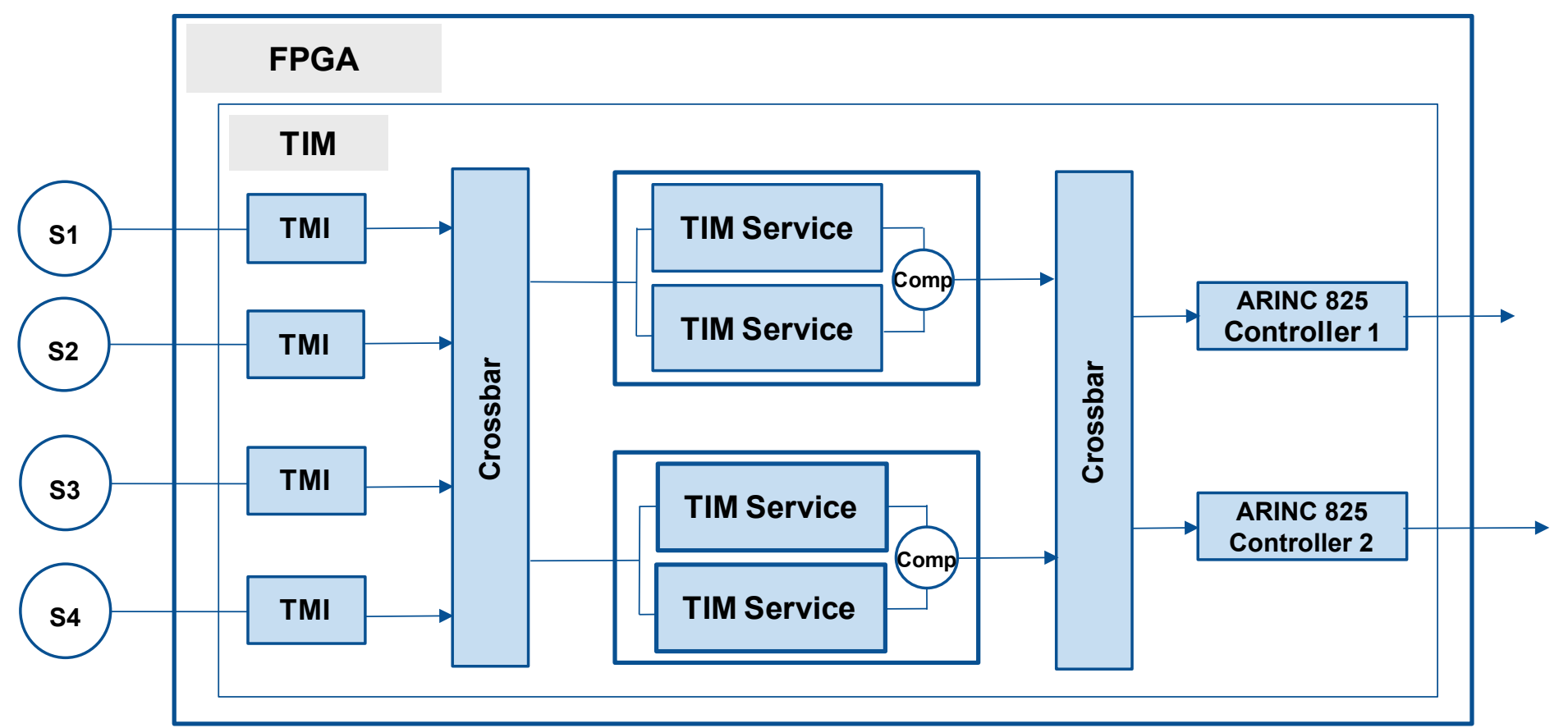

ARINC 825 bus

TIM-service cores aggregated in redundant lock-step pairs 


\section{Interfaces Design 3.3 Dual-chip Interface}

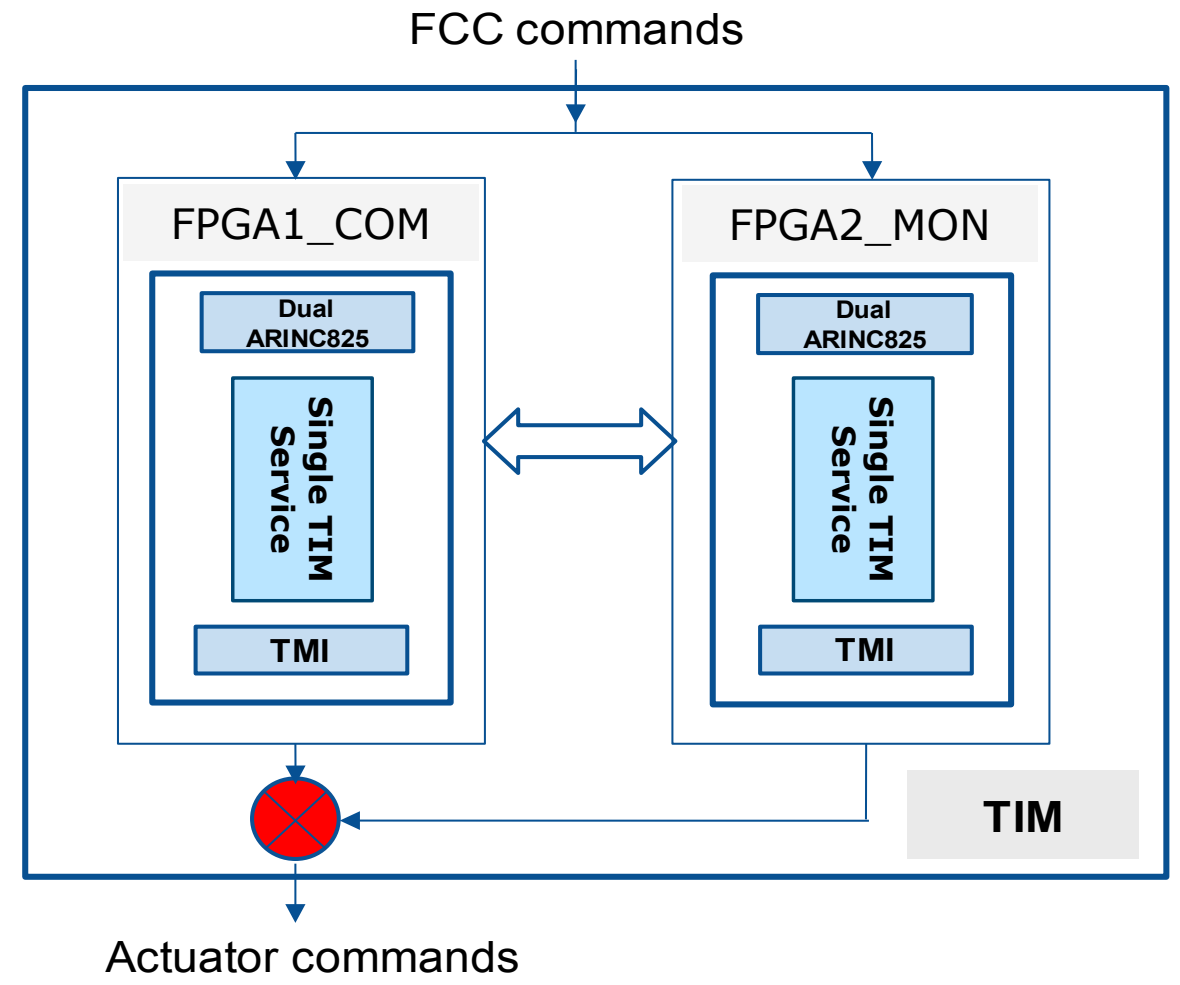

) Fault detection in ensured through the comparison of the outputs of the COM and MON lanes 


\section{Interfaces Design 3.4 Reliability and Safety Analysis}

\section{Markov Chains-based modeling}

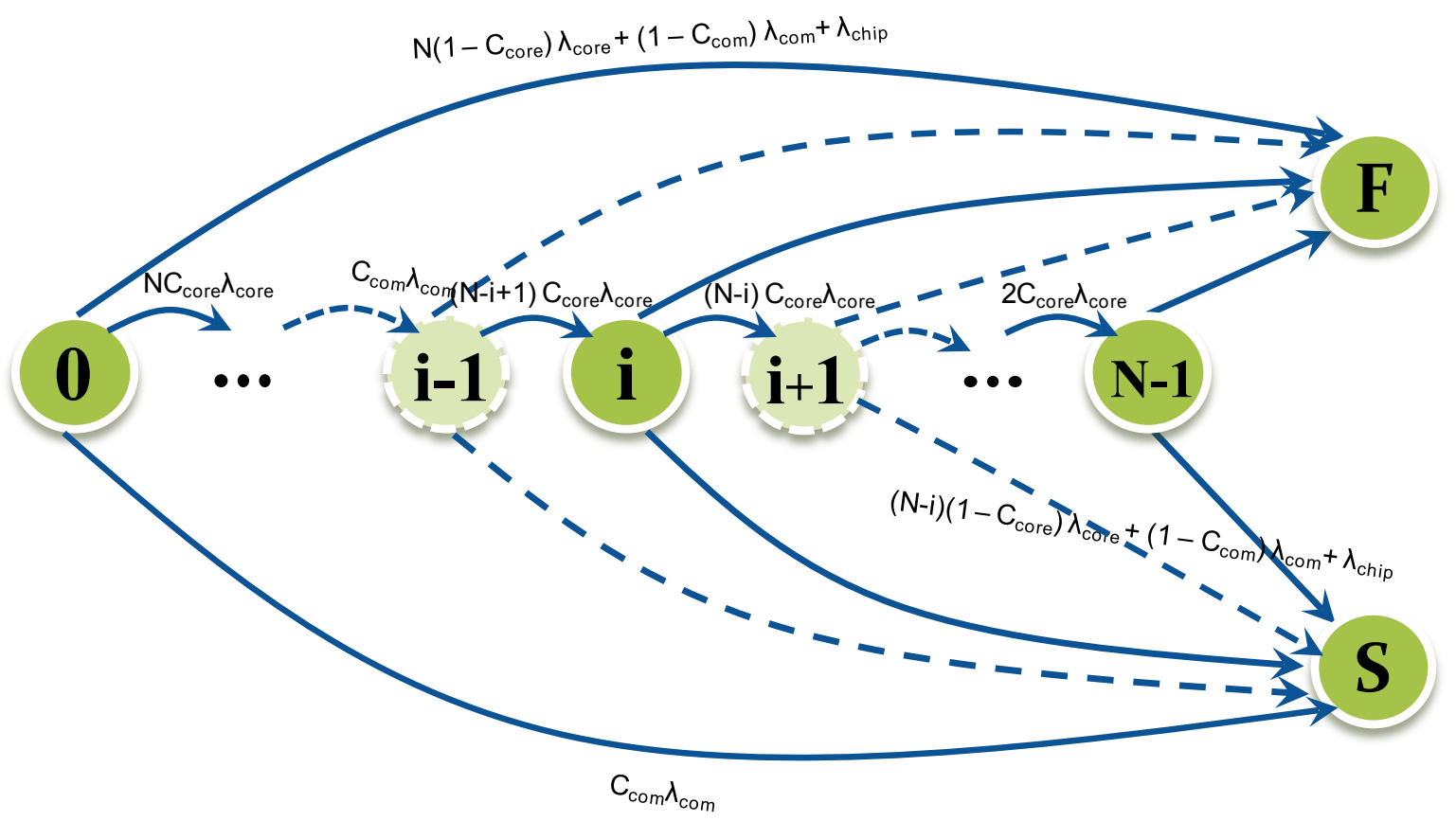

Single-chip model

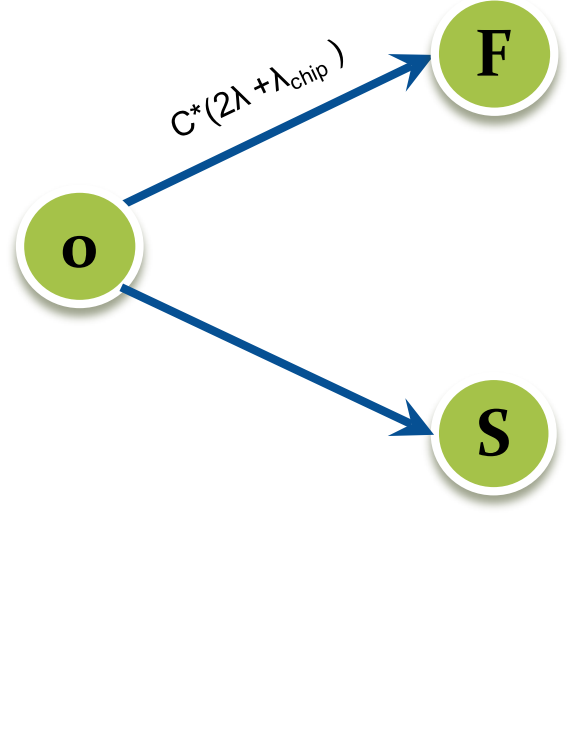

Dual-chip model 


\section{Interfaces Design 3.5 Analysis Results}

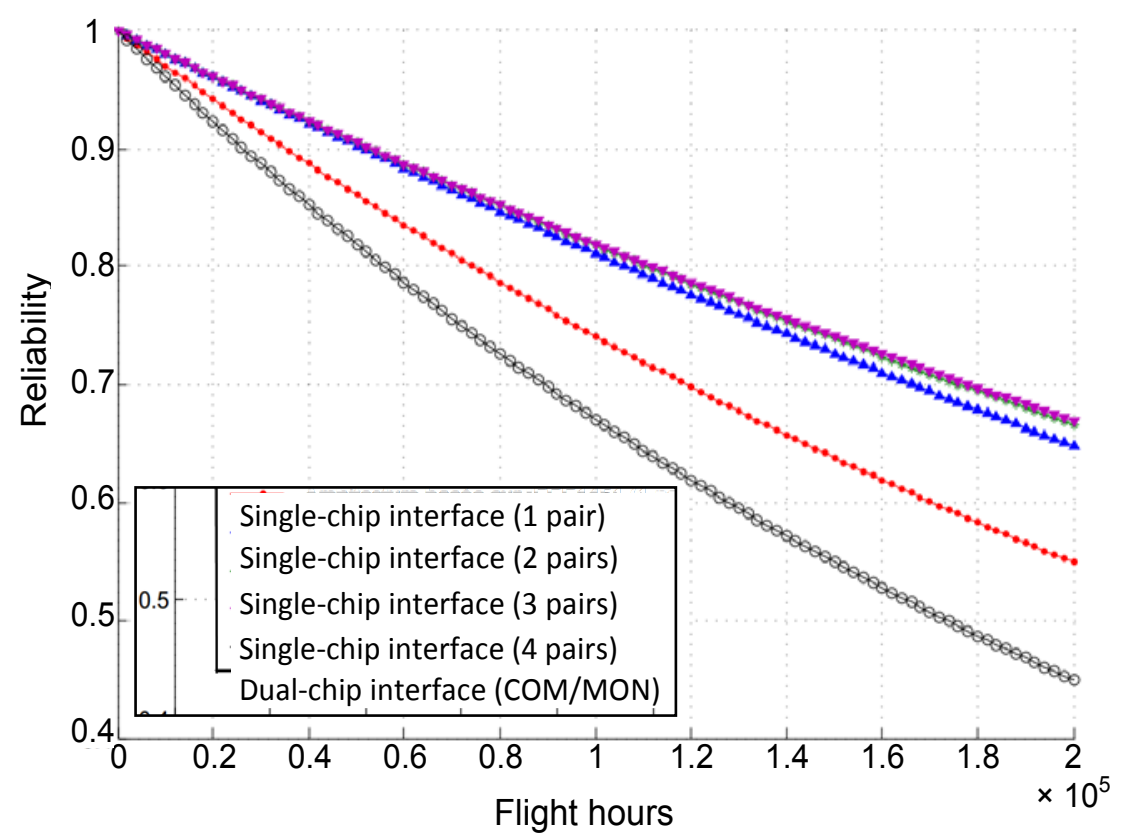

Reliability

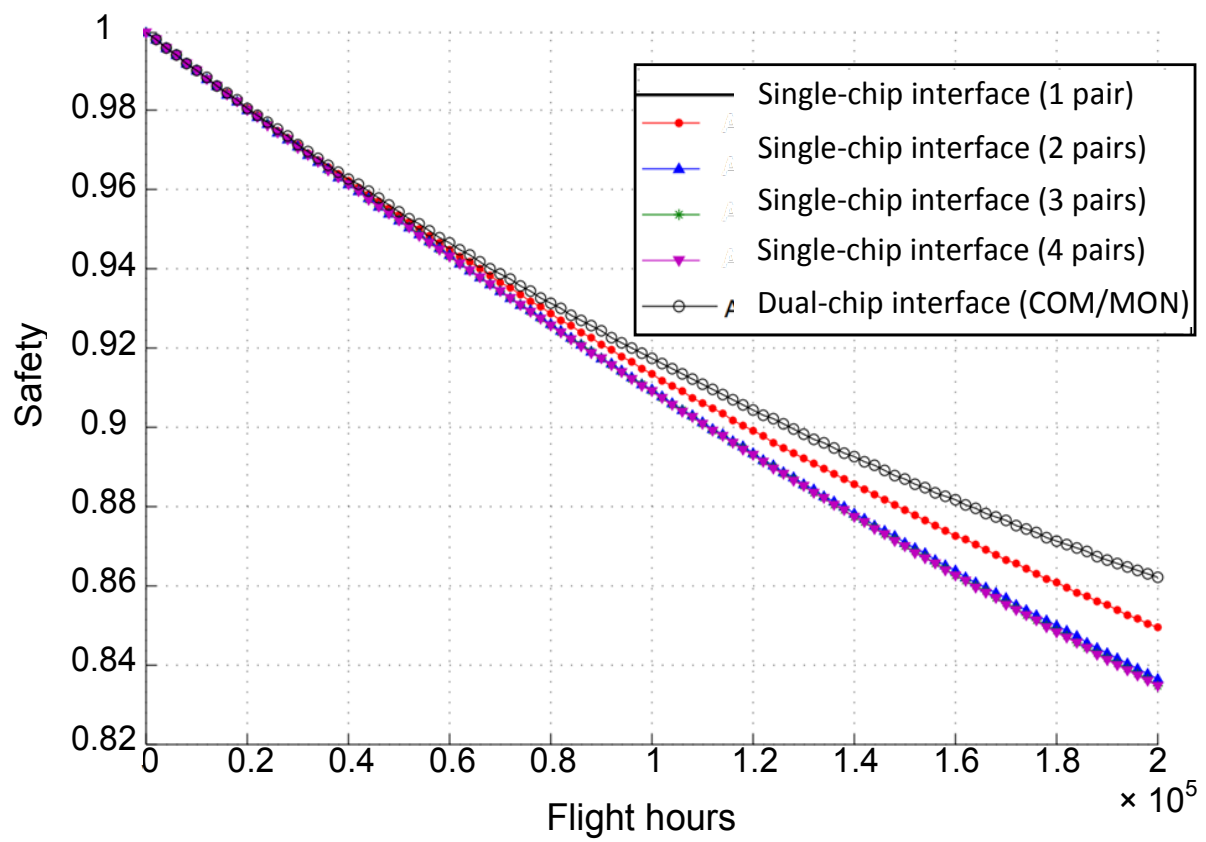

Safety 


\section{The Prototype \\ 4.1 Prototyping advantages}

\section{Prototyping allows:}

> Identifying practical challenges and constraints

) Testing and benchmarking new algorithms

) Concept validation in early development stages

) Characterizing the design

Vxposing implementation challenges 


\section{The Prototype 4.2 Prototype description (1)}

) The prototype includes

- 2 LX45T FPGA boards

-2 Mezzanine boards

- Dual ARINC 825 bus

- COTS sensors: AD7415 temperature sensors

> Configured to maintain

$-1 \mathrm{Mbit} / \mathrm{s}$ throughput

- Guaranty determinism 


\section{The Prototype 4.2 Prototype description (2)}

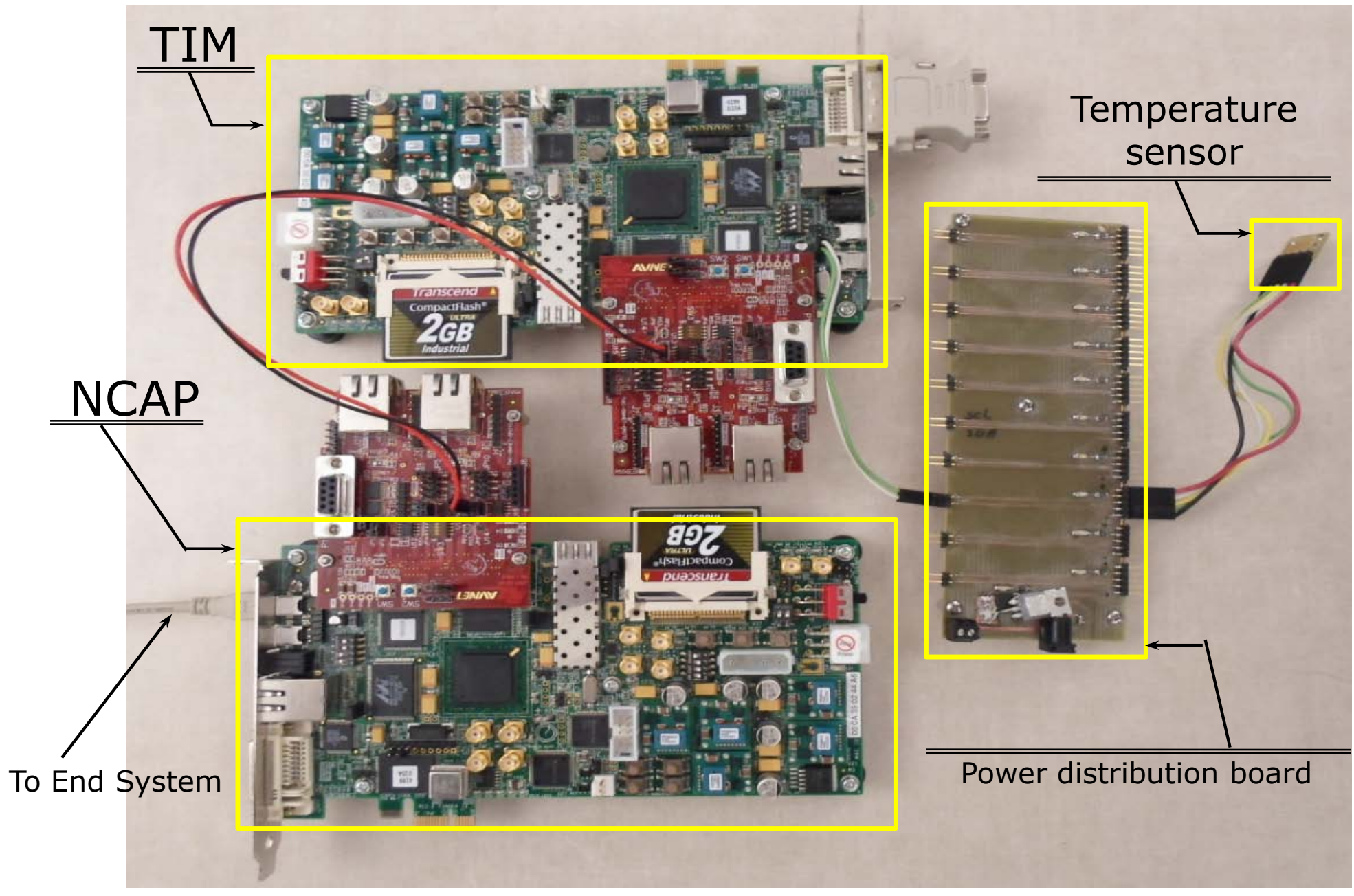




\section{The Prototype}

4.3 TIM services

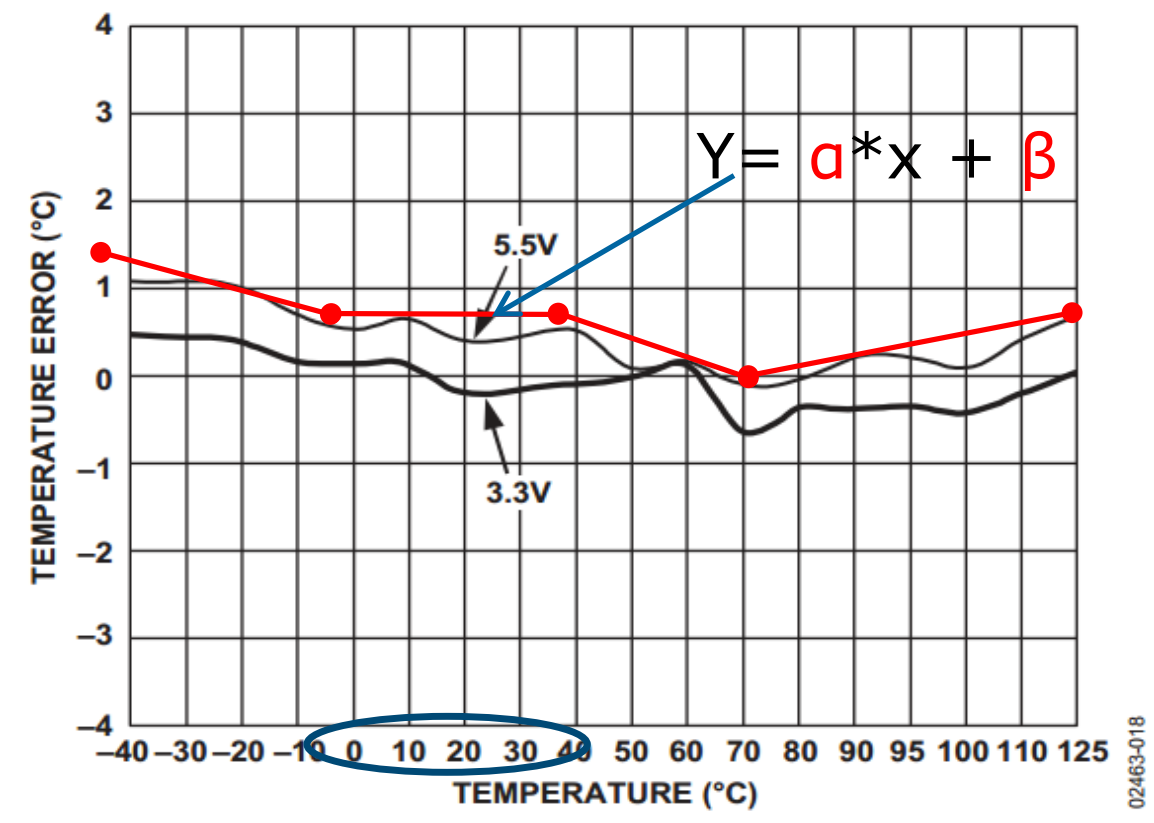

8. Typical Temperature Error @ 3.3 V and 5.5 V

Dxample of a service offered by the TIM prototype

- Data validation and correction

- Coefficients are stored within the TEDS 


\section{The Prototype \\ 4.4 NCAP}

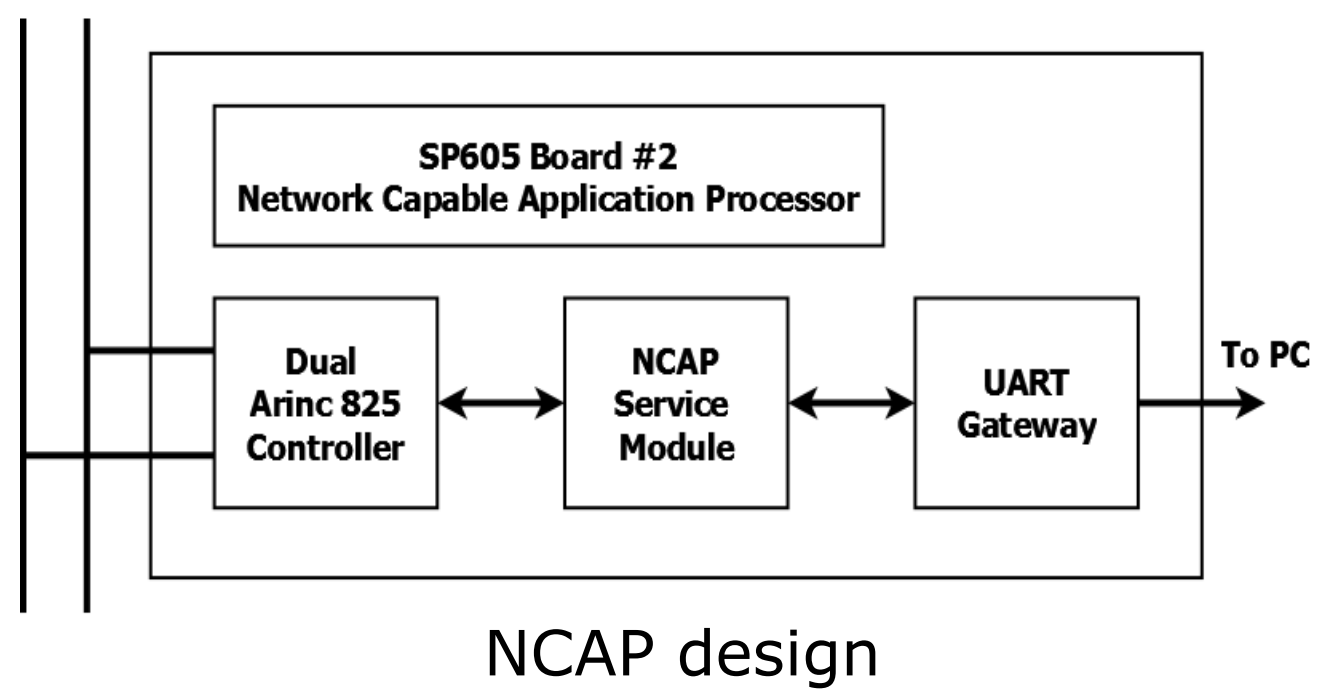

> The main functionalities of the NCAP are:

- Data flow management (ARINC 825/UART-USB)

- Data messages formatting (NCAP Service)

- Latency measurement (NCAP Service) 


\section{Test and Validation 5.1 Fault Tolerance Validation}

>bjectives:

- Evaluate the interface capacity to detect and recover from faults

- Evaluate messages latency in normal operation mode

- Evaluate the impact of occurrence of faults on messages delays.

Test procedure

- Based on fault injection technique

- Emulating the occurrence of faults in the TIM-service pairs. 


\section{Test and Validation 5.2 Latency Validation}

Latency Measurment :

- NCAP_Latency is the delay for transferring messages through the ARINC 825 bus

- Measured total latency $=$ TIM_latency + NCAP_latency

- The real latency is calculated by compensating the measured latency

Message structure

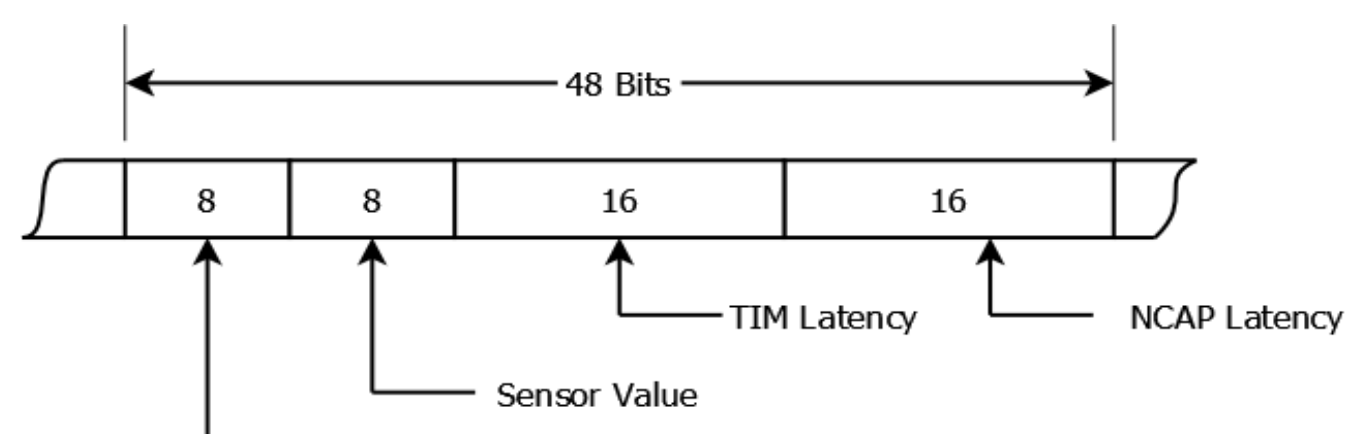

SRC, DST, Health Management 


\section{Test and Validation 5.3 Results}

\begin{tabular}{|c|c|c|c|c|}
\hline Sensor & $\begin{array}{c}\text { Normal } \\
\text { operation }(\mathrm{ms})\end{array}$ & $\begin{array}{c}1 \text { faulty pair } \\
(\mathrm{ms})\end{array}$ & $\begin{array}{c}2 \text { faulty pairs } \\
(\mathrm{ms})\end{array}$ & $\begin{array}{c}3 \text { faulty pairs } \\
(\mathrm{ms})\end{array}$ \\
\hline S1 & 0.4 & 0.4 & 0.4 & 0.4 \\
\hline S2 & 0.5 & 0.6 & 0.6 & 0.6 \\
\hline S3 & 0.6 & 0.9 & 0.9 & 0.9 \\
\hline S4 & 0.7 & 1.1 & 1.1 & 1.1 \\
\hline S5 & 0.4 & 0.4 & 0.4 & 1.4 \\
\hline S6 & 0.5 & 0.5 & 0.6 & 1.6 \\
\hline S7 & 0.6 & 0.6 & 0.9 & 1.9 \\
\hline S8 & 0.7 & 0.7 & 1.1 & 2.1 \\
\hline
\end{tabular}

Latency measures

- Fault tolerance mechanisms tested and validated

- The 2 ms message latency constraint is always satisfied 


\section{Conclusion}

\section{New smart transducers interfaces}

) Two interface designs based on IEEE 1451

Improved reliability and safety

Validated through a hardware prototype (FPGAs,COTS ...)

Integrated into a dual ARINC 825 bus

\section{Future work}

) Implementation and validation of the dual-chip interface architecture 


\section{Thank you for your attention}

